\title{
The best time for orthodontic treatment for Polish children based on skeletal age analysis in accordance to refund policy of the Polish National Health Fund (NFZ)
}

\author{
Rafał Flieger ${ }^{1, A-D}$, Jacek Matys ${ }^{2,3, C-F}$, Marzena Dominiak ${ }^{4, E, F}$ \\ ${ }^{1}$ Private Dental Practice, Kościan, Poland \\ ${ }^{2}$ Phd Student, Department of Oral Surgery, Wroclaw Medical University, Poland \\ ${ }^{3}$ Private Dental Practice, Wschowa, Poland \\ ${ }^{4}$ Department of Oral Surgery, Wroclaw Medical University, Poland
}

A - research concept and design; $\mathrm{B}$ - collection and/or assembly of data; $\mathrm{C}$ - data analysis and interpretation;

$D$ - writing the article; $E$ - critical revision of the article; $F$ - final approval of the article

\section{Rafał Flieger \\ E-mail:gabinet6@op.pl \\ Funding sources \\ None declared \\ Conflict of interest \\ None declared}

Address for correspondence

Received on June 14, 2016 Reviewed on July 12, 2016 Accepted on March 29, 2017

DOI

10.17219/acem/69976

\section{Copyright}

Copyright by Author(s)

This is an article distributed under the terms of the

Creative Commons Attribution Non-Commercial License

(http://creativecommons.org/licenses/by-nc-nd/4.0/)

\begin{abstract}
Background. The ability to estimate the growth potential correctly is an important factor in taking effective actions with respect to orthodontic diagnosis, suitable treatment protocol and optimal timing of commencing the therapy.
\end{abstract}

Objectives. The aim of this study was to compare skeletal maturity between the groups depending on the malocclusion as well as to define the optimal timing for particular orthodontic treatment procedures in children with miscellaneous types of malocclusion and compare it with the duration of treatment proposed by the National Health Fund (NFZ).

Material and methods. The delivery of the objectives of this study has been divided into 4 stages: the selection of the lateral head films ( $n=180$ ) of patients with malocclusion without congenital defect - diagnosing an orthodontic defect using Angle's classification, noting the chronological age (7-16 years) at the moment of taking the radiograph; the evaluation of the patients's skeletal maturation stages determined by the cervical vertebrae; comparison of the skeletal maturity between the groups considering the calendar age and the type of malocclusion, and analysis of 45 cephalometric RTG projections of patients with different malocclusions.

Results. The average age for children with malocclusion was significantly higher for class III as compared to class II or l in pubertal peak group (CS3 and (S4), and for class III as compared to class I or II after the puberty peak (CS5 and CS6). Our findings of the average age according to Angle's classification revealed significant differences between class II and class III at CS2, CS3, CS4, and CS5, between class I and class III at CS3 and CS5, between class I and class II at CS4, between class III and I at CS5, and between all classes at CS6 maturity stage.

Conclusions. Based on our research, it was concluded that the time of the refund of orthodontic treatment in Poland should be extended to 13.6 years of age for class $\mid 1$ malocclusion and to 14.7 years of age for class III defects.

Key words: orthodontic treatment, malocclusion, Angle's classification, skeletal age, cervical vertebral maturation method 


\section{Introduction}

The ability to estimate the growth potential correctly is an important factor in taking effective actions with respect to orthodontic diagnosis, suitable treatment protocol and optimal timing of commencing the therapy. ${ }^{1}$ The assessment of skeletal maturity in patients is essential to avoid complications related to early discontinuation of class III malocclusion therapy as well as to carry out an effective treatment with the use of removable functional appliances in children with class II deviations. ${ }^{2}$ Moreover, it has been stated that clinical effects of the therapy are observed the soonest in patients whose treatment commenced at the right time, i.e., during the peak growth. ${ }^{3}$

The most popular methods of evaluating bone age include the analysis of the cervical vertebrae in lateral head films and the radiographs of hand and wrist bones. ${ }^{4}$ Warmeling et al. also believe that, although the correlation between both methods is very high, the popularity of the method using lateral head radiographs has been growing in the recent years. ${ }^{5}$

Taiwanese scientists published a report about the significance of the biological factors (breaking of voice in boys, the pace of the bodily growth, menstruation in girls) in monitoring the growth. Based on an analysis of 304 girls, they proved that girls started menstruating ca. 1 year after the peak growth defined by means of the analyses of the hand and wrist X-rays as well as lateral head radiographs. ${ }^{6}$

The relation between skeletal maturity (relying on the maturity of the vertebrae) and dentition development has also been described in the literature. By evaluating the mineralization stages for specific teeth, the highest correlation between the shapes of certain vertebrae and the $2^{\text {nd }}$ lower molar in girls and the lower canine in boys was identified. The mineralization of teeth was also correlated with the skeletal age. ${ }^{7}$

Based on an evaluation of the shape of the cervical vertebrae and subsequent stages of the dental age in teenage patients, Gupta et al. presented treatment protocols for various malocclusion defects depending on the timing of the therapy. ${ }^{8}$ They assessed the dental age by means of Demirjian's method and the skeletal age was estimated with the use of the cervical stage method. The authors emphasized that the treatment of various types of occlusal defects should be commenced depending on the patient's skeletal age.

On the basis of our clinical experience in the field of orthodontic treatment of children and the observation of rules for granting refunds by NFZ, we came to the conclusion that the relationship between the length of the duration of the growth spurt and the type of malocclusion should be carefully investigated.

The assumption of the work was that the stages of accelerated growth (CS3-CS4) may occur at a different calendar age depending on the existing malocclusion.
The aim of this study was to compare skeletal maturity between the groups depending on the malocclusion as well as to define the optimal timing for particular orthodontic treatment procedures in children with miscellaneous types of malocclusion and compare it with the duration of treatment proposed by the National Health Fund (NFZ).

\section{Material and methods}

\section{Study population}

Research material consisted of 180 lateral head films of patients (124 women, 56 men) with different malocclusions, selected from the medical records of 350 patients treated in an orthodontic clinic between 2014 and 2016 (Table 1).

Table 1. Distribution of the patients according to stages of the skeletal development

\begin{tabular}{|l|c|c|c|}
\multicolumn{2}{|c|}{ Stages of skeletal maturity } & \multicolumn{2}{c|}{$\begin{array}{c}\text { Age of patients with } \\
\text { malocclusions [years] }\end{array}$} \\
\hline \multicolumn{1}{|c|}{ CS } & $n$ & mean & SD \\
\hline CS1 & 30 & 8.4 & 1.8 \\
\hline CS2 & 30 & 9.5 & 1.2 \\
\hline CS3 & 30 & 11 & 1.6 \\
\hline CS4 & 30 & 12.6 & 1.7 \\
\hline CS5 & 30 & 12.9 & 0.8 \\
\hline CS6 & 30 & 14.8 & 1.1 \\
\hline Total & 180 & 11.5 & 1.9 \\
\hline
\end{tabular}

\section{Study protocol}

According to Angle's definition, in class I malocclusions, the mesiobuccal cusp of the maxillary $1^{\text {st }}$ molar occludes with the buccal grove of the mandibular $1^{\text {st }}$ molar. Irregularity consists in the disorders in the anterior section relative to the midline or horizontal plane; it may also involve Angle's class II malocclusion, which covers distal occlusion and includes 2 divisions. In class II div. 1, the mesiobuccal cusp of the maxillary $1^{\text {st }}$ molar is located anteriorly to the buccal grove of the mandibular $1^{\text {st }}$ molar. The interincisal angle is decreased, which is related to the proclination of the incisors (protrusion). In this group, the increase of the overjet above $3 \mathrm{~mm}$ is also observed. In class II div. 2, the interincisal angle is increased due to the retrusion of the incisors, which may compensate for the increased overjet. In Angle's class II, the radiographs of patients with incisal protrusion and retrusion were analyzed collectively.

In class III, the mesiobuccally cusp of the maxillary $1^{\text {st }}$ molar is located posteriorly to the buccal grove of the mandibular $1^{\text {st }}$ molar. 
The study has been divided into 4 stages:

I. The selection of lateral head films of patients with malocclusion without congenital defects:

- diagnosed Angle's I, II or III class orthodontic defect;

- chronological age of 7-16 years at the moment of taking the radiograph.

II. The assessment of skeletal maturity in 3 groups.

III. The comparison of the skeletal maturity between the groups considering the calendar age and the type of malocclusion - statistical analysis.

IV. The analysis of 45 cephalometric RTG projections of patients at the calendar age of 7-16 years with different malocclusions to assess skeletal maturity. The study used the CS method presented by Franchi et al. and also described by Flieger et al. ${ }^{3,9}$ :

- CS1: the bottom edges of the $2^{\text {nd }}$ and $3^{\text {rd }}$ vertebrae are straight, the $3^{\text {rd }}$ and $4^{\text {th }}$ vertebrae have a trapezoidal shape - growth acceleration will occur in 2 years;

- CS2: the bottom edge of the $2^{\text {nd }}$ vertebra becomes concave, the inferior edges of the of $3^{\text {rd }}$ and $4^{\text {th }}$ vertebrae are straight; a trapezoid shape of the $3^{\text {rd }}$ and $4^{\text {th }}$ vertebrae can be noticed - growth acceleration will occur in ca. 1 year;

- CS3: the inferior edges of the $2^{\text {nd }}$ and $3^{\text {rd }}$ vertebrae are concave, a more rectangular shape of the $2^{\text {nd }}$ and $3^{\text {rd }}$ vertebrae occurs - the growth peak began last year;

- CS4: the concavity is visible in the bottom edges of the $2^{\text {nd }}, 3^{\text {rd }}$ and $4^{\text {th }}$ vertebrae, a rectangular shape of the $3^{\text {rd }}$ and $4^{\text {th }}$ vertebrae begins to be seen in a horizontal plane - the growth peak has begun;

- CS5: the concavity is visible in the bottom edges of the $2^{\text {nd }}, 3^{\text {rd }}$ and $4^{\text {th }}$ vertebrae, a square shape of the $3^{\text {rd }}$ and $4^{\text {th }}$ vertebrae begins to be seen - the growth peak ended a year before that stage;

- CS6: strongly visible concavities on the bottom edges of the $2^{\text {nd }}, 3^{\text {rd }}$ and $4^{\text {th }}$ vertebrae can be noticed, a rectangular shape of the $3^{\text {rd }}$ and $4^{\text {th }}$ vertebrae begins to be seen in a vertical plane - the growth peak ended 2 years before that stage.

\section{Statistical analysis}

The statistical analysis was conducted using STATISTICA v. 9.0 software (StatSoft Inc., Tulsa, USA). The average chronological age of the patients with malocclusions and their skeletal age was compared between the groups using the one-way ANOVA test. Pair comparisons were carried out based on the Tukey's post hoc test at a significance level $\mathrm{p}=0.05$.

\section{Results}

An analysis of the average age according to Angle's classification revealed no significant differences between class II and class III at CS1 and CS2 maturity stages (Table 2).
Table 2. An analysis of the average age according to the Angle's classification at CS1 and CS2 maturity stages

\begin{tabular}{|l|c|c|c|c|}
\multicolumn{3}{|c|}{ CS1 and CS2 } & \multicolumn{2}{c|}{ Age } \\
\hline Angle's class & $n$ & $\%$ & mean & SD \\
\hline I & 0 & 0.0 & - & - \\
\hline II & 29 & 48.3 & 8.3 & 0.74 \\
\hline III & 31 & 51.7 & 8.1 & 0.34 \\
\hline Total & 60 & 100 & 8.2 & 0.57 \\
\hline
\end{tabular}

II vs II: $p>0.05$

The mean age of the subjects with class III malocclusion in pubertal peak group (CS3 and CS4) was significantly higher as compared to class II or class I (Table 3).

Table 3. An analysis of the average age according to the Angle's classification at CS3 and CS4 maturity stages

\begin{tabular}{|l|c|c|c|c|}
\multicolumn{3}{|c|}{ CS3 or CS4 } & \multicolumn{2}{c|}{ Age } \\
\hline Angle's class & $\mathrm{n}$ & $\%$ & mean & SD \\
\hline I & 22 & 36.6 & 11.8 & 1.33 \\
\hline II & 23 & 38.3 & 11.2 & 1.27 \\
\hline III & 15 & 25.1 & 12.8 & 0.48 \\
\hline Total & 60 & 100 & 11.8 & 1.29 \\
\hline
\end{tabular}

II vs II: $p<0.05 ;$ I vs III: $p<0.05$; | vs II: $p>0.05$

Our findings show that patients with class III malocclusions as compared to class I according to Angle's classification commence the CS5 and CS6 phase of the skeletal development significantly later.

Table 4. An analysis of the average age according to the Angle's classification at CS5 and CS6 maturity stages

\begin{tabular}{|l|c|c|c|c|}
\multicolumn{3}{|c|}{ CS5 and CS6 } & \multicolumn{2}{c|}{ Age } \\
\hline Angle's class & $n$ & $\%$ & mean & SD \\
\hline I & 21 & 35 & 12.9 & 0.88 \\
\hline$\|$ & 19 & 32 & 13.6 & 1.28 \\
\hline III & 20 & 33 & 14.7 & 0.95 \\
\hline Total & 60 & 100 & 13.8 & 1.30 \\
\hline
\end{tabular}

II vs III: $p<0.05$; I vs III: $p<0.002$; I vs II: $p>0.05$

An analysis of the average age according to Angle's classification revealed significant differences between class II (mean $9.2 \pm 0.29$ years) and class III (mean $8.53 \pm 0.17$ years) at CS2 maturity stage (Fig. 1).

An analysis of the average age according to Angle's classification revealed significant differences between class III (mean $10.87 \pm 1.07$ years) and class I (mean $10.44 \pm 0.98$ years) or class II (mean $12.37 \pm 0.14$ years) at CS3 maturity stage (Fig. 2).

An analysis of the average age according to Angle's classification revealed significant differences between 
class I (mean $13.25 \pm 0.31$ years) and class II (mean $12.10 \pm 0.95$ years) or class II and class III (mean $13.29 \pm 0.10$ years) at CS4 maturity stage (Fig. 3).

An analysis of the average age according to Angle's classification revealed significant differences between class I (mean $12.46 \pm 0.61$ years) and class III (mean $13.56 \pm 0.26$ years) or class II (mean $12.63 \pm 0.61$ years) and class III at CS5 maturity stage(Fig. 4).

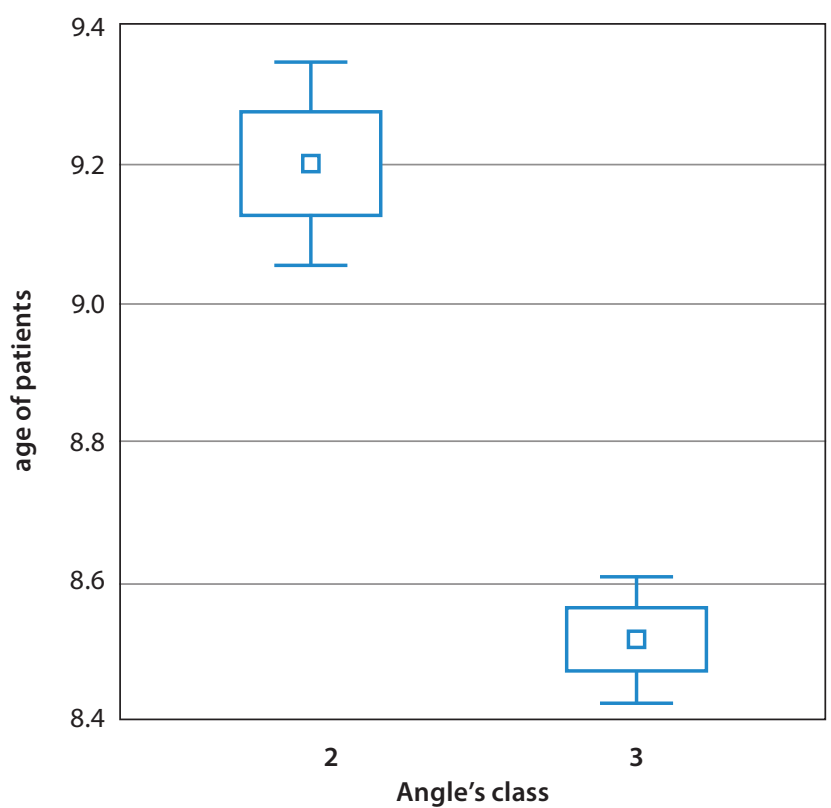

Fig. 1. An analysis of an average age according to the Angle's classification at CS2 maturity stage

II vs III: $p<0.0002$.

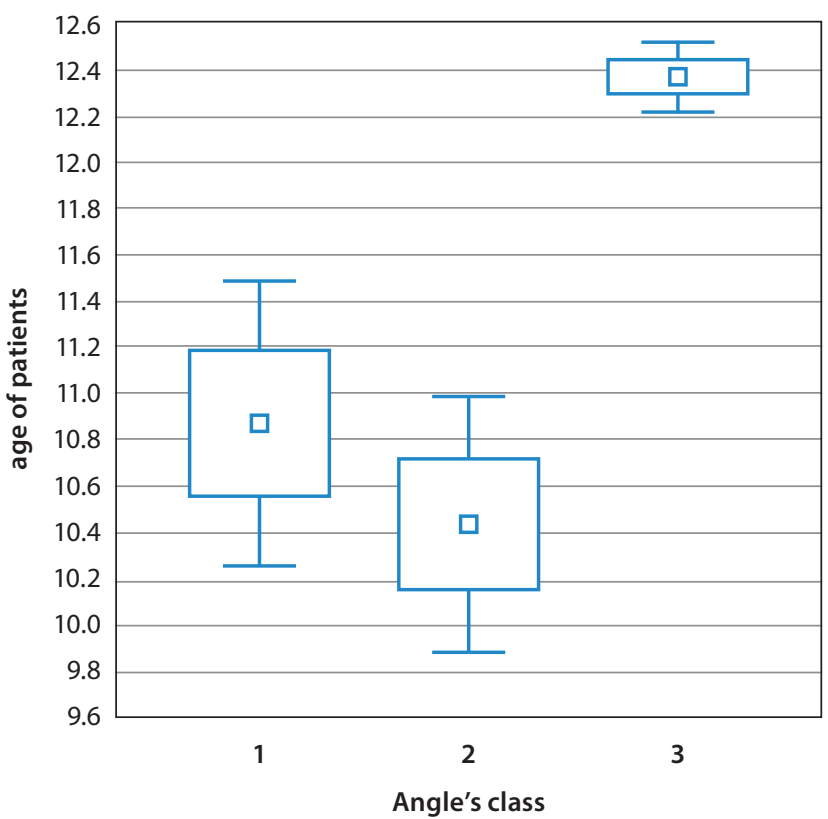

Fig. 2. An analysis of an average age according to the Angle's classification at CS3 maturity stage

II vs III: $p<0.0004$; I vs III: $p<0.0004$
Furthermore, significant differences in the patients' average age at CS6 maturity stage between all classes of malocclusion according to Angle's classification were noted. The average age of the subjects with class III malocclusions was significantly higher as compared to class II and class I, as well as for class II as compared to class I.

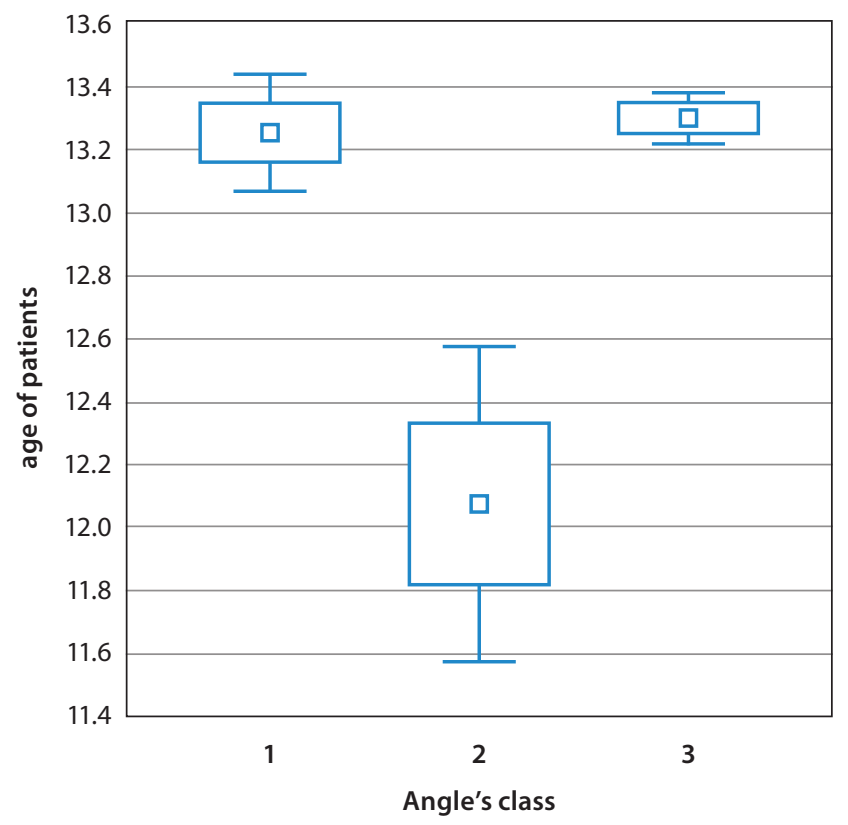

Fig. 3. An analysis of an average age according to the Angle's classification at CS4 maturity stage

II vs II: $p<0.0001 ;$ II vs III: $p<0.0001$.

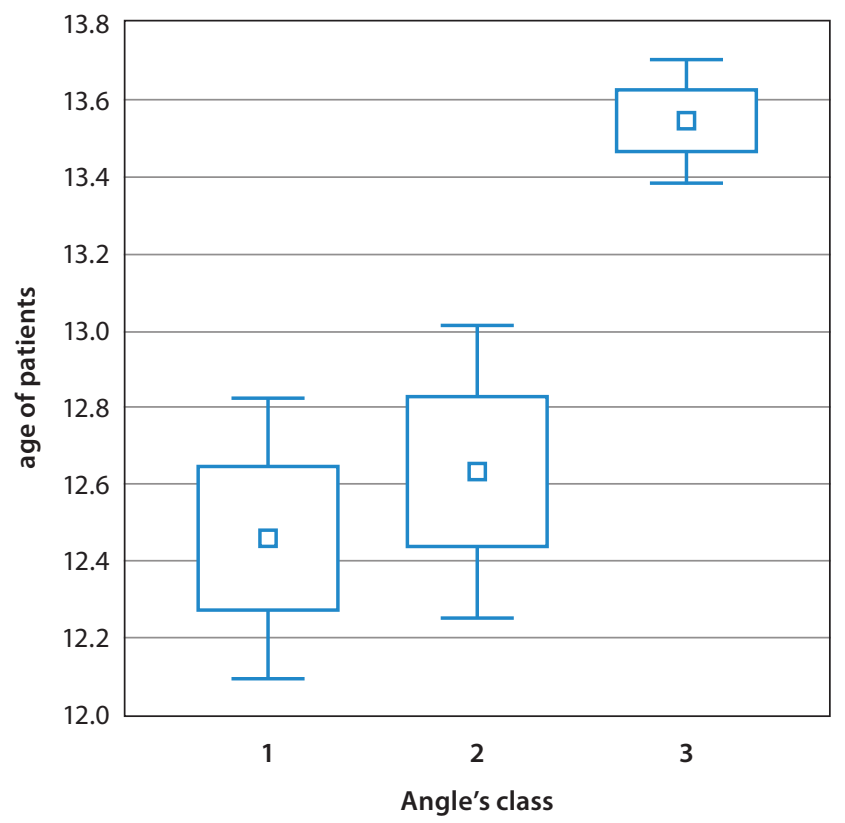

Fig. 4. An analysis of an average age according to the Angle's classification at CS5 maturity stage

| vs III: $p<0.0003$; || vs |||: $p<0.002$. 


\section{Discussion}

The analysis of the mandibular growth potential, in particular as regards the diagnosis of Angle's class III malocclusion, is an extremely important factor in the effective planning of the orthodontic therapy at different levels of an individual's development. ${ }^{10}$ Chen et al. evaluated the change in the length of the mandible at particular stages of the skeletal development based on the analysis of the shape of the cervical vertebrae. ${ }^{11}$ The length of the mandible was examined by them on lateral head films by measuring the distance between the points Ar and Pog at stages CS1 and CS4, i.e., from 2 years before the peak growth to the total completion of the growth. Researchers observed that the distance obtained at stage CS4 was taken away from the distance obtained at stage CS1. In that manner, the authors created a system allowing us to predict the actual value of the mandibular growth based on the evaluation of the maturity of the cervical vertebrae and, therefore, make better use of specific types of orthodontic appliances in the treatment of various types of malocclusions.

Our study also allows for effective planning of orthodontic treatment in Polish children with various malocclusions based on ascertaining the skeletal age in terms of the chronological age and reimbursement needs.

Kuc-Michalska and Baccetti studied the length of the peak growth in patients with Angle's class I and III defects. ${ }^{12}$ Based on the analysis of 218 lateral head films (93 women and 125 men) taken between stages CS3 and CS4 of the peak growth, the authors demonstrated that the peak growth occurs at a similar chronological age in patients with Angle's class I and III. However, they also observed that the time interval between stages CS3 and CS4 in patients with class I was 11 months vs 16 months for class III patients.

According to our research, the average age of the patients with class I and III malocclusions during the growth spurt demonstrate the difference of 12 months in favor of class III. The time interval was not tested, because patients with the skeletal age at stages CS3 and CS4 were counted as 1 group.

Other authors suggested that a greater growth of the mandible length in patients with Angle's class III malocclusions was related to a longer duration of adolescence. ${ }^{13}$ Other sources indicate that the treatment of class III malocclusions should start as early as possible, optimally right after the diagnosis. ${ }^{14}$

By measuring the difference between the chronological age of the patients after the puberty peak (CS5 and CS6) and the patients before the puberty peak (CS1 and CS2), we showed the highest difference amounting to 6.6 years, recorded for children with class III malocclusions. Thus, we demonstrated that the adolescence period in this type of malocclusion takes the longest and ends at around 14 years of age. Furthermore, we showed that the patients with class III defects appeared in the treatment with an average age of 8.1 years, which seems to be a good time to start orthodontic treatment of this malocclusion.
Baccetti emphasized the importance of predicting the growth peak for higher effectiveness of treating Angle's class II malocclusion. ${ }^{15} \mathrm{He}$ demonstrated that functional therapies of distal occlusion (e.g., with a Twin-block appliance) are the most effective when they commence at the end of stage CS2 or at the beginning of stage CS3. In his opinion that allows for the reduction of the treatment time to the necessary minimum.

In our study group, we noticed that the average age for children with class II malocclusion in pubertal peak group (CS 3 and CS4) was 11.2 years. By measuring the difference between the chronological age of class II patients after the puberty peak (CS5 and CS6) and patients before the puberty peak (CS1 and CS2), we showed a difference amounting to 5.3 years. This means that in patients with this type of malocclusion, adolescence lasts about 1 year shorter than in patients with class III malocclusions.

Simultaneously, the average of the chronological age for class II and III malocclusion patients was similar at stages CS1 and CS2, while being higher at stages CS5 and CS6. This suggests that the growth acceleration lasts longer in patients with diagnosed class III malocclusions than in patients with class II.

Klimas et al. conducted a study which aimed to estimate the bone age by the analysis of the cervical vertebrae on the cephalograms (CVM) in Polish children with standard occlusion and children with hypodontia. ${ }^{16}$ The patients were classified into 2 groups: a group of patients with standard occlusion and a group with shortages of permanent tooth buds. The skeletal age of children from both groups was estimated. Then, skeletal maturity was compared between the groups, taking into consideration the similar calendar age of the patients. The authors proved that the CVM method enables an effective assessment of the skeletal age and a demonstration of the differences between the calendar age and bone maturity. Furthermore, they observed a delay in reaching skeletal maturity by children with congenital missing teeth compared to children with standard occlusion. ${ }^{16}$

Our assessments and analysis of the skeletal age enabled us to successfully determine the growth stage of the patients with different types of malocclusion, which allows for a highly effective orthodontic therapy in Polish children, and may be an indication of the National Health Fund's refund rules.

\section{Conclusions}

It was shown that the accelerated growth stage (CS3CS4) can appear at a different age depending on the type of malocclusion. This information is very important in regards to the beginning of the treatment of this type of malocclusions.

Accelerated growth (the best for class II therapy) occurs at the average age of 11.2 years. In the same period, class III patients show the average age of 12.8. A period 
of accelerated growth takes longer in patients with class III than in patients with class II. According to Angle, the difference in time reaches about 1 year and 6 months. The expiry of the growth spurt occurs around the age of 13 years for all types of malocclusions.

Based on our research, it is concluded that the time of the refund of orthodontic treatment in Poland should be extended to 13.6 years of age for class II malocclusions and to 14.7 years of age for class III defects.

\section{References}

1. Sato K, Mito T, Mitani H. An accurate method of predicting mandibular growth potential based on bone maturity. Am J Orthod Dentofacial Orthop. 2001;120:286-293.

2. Sato K, Mito T, Mitani H. Predicting mandibular growth potential with cervical vertebral bone age. Am J Orthod Dentofacial Orthop. 2003;124:173-177.

3. Franchi L, Baccetti T, De Toffol L, Polimeni A, Cozzad P. Phases of the dentition for the assessment of skeletal maturity: A diagnostic performance study. Am JOrthod Dentofacial Orthop. 2008;133:395-400.

4. Uysal T, Ramoglu SI, Basciftci FA, Saric Z. Chronologic age and skeletal maturation of the cervical vertebrae and hand-wrist: Is there a relationship? Am J Orthod Dentofacial Orthop. 2006;130:622-628.

5. Warmeling D, Rodrigues KM, Zastrow MD, Thiesen G. Skeletal maturation evaluation indexes. Odonto Cienc. 2010;25:188-193.

6. Lai HH, Chang ZC, Yao CC, et al. Relationship between age at menarche and skeletal maturation stages in Taiwanese female orthodontic patients. Form Med Assoc. 2008;107:527-532.

7. Chen J, Hu H, Guo J, et al. Correlation between dental maturity and cervical vertebral maturity. Oral Surg Oral Med Oral Pathol Oral Radiol Endod. 2010;110:777-783.

8. Gupta KP, Garg S, Grewal PS. Establishing a diagnostic tool for assessing optimal treatment timing in Indian children with developing malocclusions. Clin Exp Dent. 2011;3(1):18-24.

9. Flieger R, Kopczyńki P, Matthews-Brzozowska T. Analysis of skeletal maturity of children with cleft palate in terms on healthy children by the CVM method. Adv Clin Exp Med. 2015;24:99-102.

10. Chen F, Terada K, Hanada K. A new method of predicting mandibular length increment on the basis of cervical vertebrae. Angle Orthod. 2004;74:630-634.

11. Chen F, Terada K, Hanada K. A special method of predicting mandibular growth potential for class III malocclusion. Angle Orthod. 2005;75:191-195.

12. Kuc-Michalska M, Baccetti T. Duration of the pubertal peak in skeletal class I and class III subjects. Angle Orthod. 2010;80:54-57.

13. Almeida MR, Almeida RR, Navarro PVPO, Conti ACCF, Navarro RL, Camacho JGDD. Early treatment of class III malocclusion: 10-year clinical follow-up. J App/ Oral Sci. 2011;19:431-439.

14. Tausche E, Luck O, Harzer W. Prevalence of malocclusions in the early mixed dentition and orthodontic treatment need. Eur J Orthod. 2004;26:237-241.

15. Baccetti T. Malocclusions de classe II: Bien choisir le moment du traitement pour optimiser l'effet orthopédique des appareils fonctionnels. Orthod Fr. 2010;81:279-286.

16. Klimas Z, Nawotczyński M, Flieger R. The diagnostics of skeletal maturation in children with standard occlusion and children with hypodontia [in Polish]. Now Lek. 2010;79:163-166. 